\title{
The fate of systemically administrated allogeneic mesenchymal stem cells in mouse femoral fracture healing
}

\author{
Shuo Huang ${ }^{1,2+}$, Liangliang $\mathrm{Xu}^{1,2+}$, Yuxin Sun ${ }^{1,2}$, Yifeng Zhang ${ }^{1}$ and Gang Li $\mathrm{i}^{1,2,3,4,5^{*}}$
}

\begin{abstract}
Introduction: The fate and whereabouts of the allogenic mesenchymal stem cells (MSCs) following their transplantation are not well understood. The present study investigated the fate of systemically administrated allogeneic MSCs in mouse fracture healing by using in vivo imaging and immunohistochemistry methods.

Methods: Open femoral fracture with internal fixation was established in 30 FVB mice, which were assigned to three groups receiving phosphate-buffered saline (PBS) injection, MSC systemic injection, or MSC local injection. Luc-MSCs $\left(5 \times 10^{5}\right)$ isolated from the luciferase transgenic mice with FVB background were injected at 4 days after fracture. All animals were terminated at 5 weeks after fracture; examinations included bioluminescence-based in vivo imaging, micro-computer tomography, mechanical testing, histology, immunohistochemistry, and double immunofluorescence staining.

Results: The bioluminescence signals of the Luc-MSCs at the fracture site could be detected for 12-14 days following their injection in the Luc-MSC local injection group, whereas in the Luc-MSC systemic injection group, LuC-MSCs were initially trapped in lungs for about 8-9 days and then gradually redistributed to the fracture site. Bone mineral density, bone volume/tissue volume, ultimate load, and E-modulus in the MSC injection groups were significantly higher than those in the PBS group. Double immunostaining demonstrated that the MSC local injection group had more Luc-positive cells, and there was a higher apoptotic rate at the fracture site than the MSC systemic injection group. Both Luciferase-positive MSCs and osteoblasts were present in the callus in the MSC injection groups at 5 weeks after fracture, suggesting that some of allogenic Luc-MSCs contributed to the new bone formation. Only less than $3 \%$ of injected Luc-MSCs remained at the fracture site in the MSC injection groups at 5 weeks following the fracture, and the rest of the injected Luc-MSCs disappeared.
\end{abstract}

Conclusions: Our data showed that both systemic and local injection of allogeneic MSCs promoted fracture healing through enhancing biomechanical properties, bone content, and enlarged callus sizes. Immunohistochemistry confirmed that the injected MSCs are still present in the fracture site and can differentiate into osteoblasts to participate in fracture healing even at 5 weeks following the fracture. These findings provide useful information for the use of allogenic MSCs for cell therapy applications.

Keywords: Allogeneic mesenchymal stem cells (MSCs), systemic injection, local injection, fracture healing

\footnotetext{
* Correspondence: gangli@cuhk.edu.hk

${ }^{\dagger}$ Equal contributors

'Department of Orthopaedics \& Traumatology, Faculty of Medicine, Room 904, 9/F, Li Ka Shing Institute of Health Institute, Prince of Wales Hospital, The Chinese University of Hong Kong, Shatin, 30-32 Ngan Shing Street, Shatin, NT, Hong Kong, SAR, PR China

${ }^{2}$ Stem Cells and Regenerative Medicine Laboratory, Li Ka Shing Institute of Health Sciences, The Chinese University of Hong Kong, Prince of Wales Hospital, 30-32 Ngan Shing Street, Shatin, NT, Hong Kong, PR China Full list of author information is available at the end of the article
} 


\section{Introduction}

Mesenchymal stem cells (MSCs), also known as multipotent mesenchymal stromal cells, have the innate ability to self-renew and differentiate into multiple cell types such as neurons, osteoblasts, cardiomyocytes, adipocytes, and chondrocytes when exposed to proper stimuli $[1,2]$, and MSCs applied locally have been used for treatments of various diseases [3-6]. Compelling evidence shows that MSC local application could repair tissue defects [7-9] and that MSCs are a promising cell source for tissue engineering $[10,11]$.

Although the use of autologous MSCs for cell therapy has been well established and accepted, the need of prolonged time for MSC culture to obtain therapeutic dose and the narrow time window for their optimal applications prohibit their wider applications. Thus, allogenic MSCs become an ideal alternative as they can be well prepared in advance, banked, and made readily available for use. However, the function and fate of allogeneic MSCs in vivo are still not well defined. Intravenous delivery of allogenic MSCs results in their specific migration to sites of injury and improves recovery in animal models of skin injury [12], stroke, and myocardial infarction [13-16]. In 2005, Shirley et al. reported that there was a systemic mobilization and recruitment of osteoblastic precursors to the fracture site via the peripheral circulation [17]. Caplan et al. also reported that MSCs delivered systemically via the circulatory system can home to target sites [18]. Taken together, allogenic MSCs applied locally and systemically could promote tissue (fracture) healing regeneration.

However, the function and fate of allogeneic MSCs in vivo are still not well defined. Some reports supported that MSCs mediate tissue and organ repair by replacing damaged cells $[19,20]$, and other studies suggest that allogeneic MSCs mainly play immune-modulatory roles in vivo [21-23]. Le Blanc et al. showed that MSCs could suppress the proliferation of both $\mathrm{CD} 4^{+}$and $\mathrm{CD} 8^{+} \mathrm{T}$ cells by upregulating the release of soluble factors such as interleukin-10 and prostaglandin $E_{2}$ [24]. It was also reported that allogeneic MSCs encouraged repair through the production of trophic factors, cytokines, and antioxidants [25-27]. Kellie et al. also found that MSC treatment increased the tensile strength of wounds and increased production and deposition of collagens in the wound [28].

There are still issues of allogenic MSC application that need further investigation: What is the fate of the allogenic MSCs in vivo? How long they can function and survive in vivo? Is there any potential immunogenic effects caused by the allogenic MSCs? In the present study, we investigated the fate and effects of systemically administrated allogeneic MSCs versus local administration of allogenic MSCs in a mouse fracture healing model.

\section{Methods}

\section{Chemicals}

The chemicals used were all purchased from SigmaAldrich (St. Louis, MO, USA) except where specified.

\section{Animal details}

Bone marrow-derived Luc-MSCs were isolated from 4week-old female CMV-luc mice (FVB/N background; Xenogen Corporation, now part of Caliper Life Sciences, Hopkinton, MA, USA). Thirty FVB/N male mice (8 weeks old, body weight of 25-35 g) were used for fracture study. All mice were housed in a designated, government-approved animal facility at the Chinese University of Hong Kong in accordance with the Chinese University of Hong Kong animal experimental regulations, and all animal experiments were approved by the Animal Research Ethics Committee of the authors' institution.

\section{Isolation and cultivation of mouse bone marrow-derived Luc-MSCs}

The CMV-luc mouse was humanely terminated. Both tibias and femurs were excised, muscle and connective tissues were removed, and then bones were stored on ice in phosphate-buffered saline (PBS) with $1 \%$ penicillinstreptomycin-neomycin (PSN). Under the laminar flow in a biological safety cabinet, two ends of bone were excised, and the marrow cavity was repeatedly flushed by $5 \mathrm{ml}$ of alpha complete culture medium (with $15 \%$ fetal bovine serum, $1 \%$ PSN). All of the bone pieces were removed from the $100-\mathrm{mm}$ culture dish by forceps and then incubated at $37{ }^{\circ} \mathrm{C}$ in a $5 \% \mathrm{CO}_{2}$ incubator for 5 days. The initial spindle-shaped cells appeared on day 3 under phase-contrast microscopy and then reached 70-90\% confluence within 2 days. Cells were trypsinized and replated by splitting at a ratio of 1:3 into new dishes. MSCs between passages 4-8 were used in this study. MSC markers CD44 and CD90, endothelial cell marker CD31, and hematopoietic marker CD45 were examined by flow cytometry in accordance with a previously published article [26]. The osteogenic and adipogenic differentiation abilities were characterized by Alizarin red and Oil red $\mathrm{O}$ staining after the corresponding inductions in accordance with our previously published article [29].

\section{Animal surgery}

A mouse open transverse femoral fracture model with internal fixation was used. In brief, the mice were under general anesthesia and sterile conditions, and a lateral incision through shaved skin and fascia lata from the left knee to the greater trochanter was made. The plane between the vasti and hamstrings was then opened by blunt dissection to expose the femur. The exact centralization of the transverse osteotomy was made by hand saw. A small incision was opened at the knee 
level, and a hole was drilled at the inter-condylar notch by using a 23-gauge hypodermic needle. A custommade stainless pin (diameter of $0.7 \mathrm{~mm}$ ) was inserted into the right femoral bone marrow cavity at the knee level to fix the fracture. The incision was closed, and a radiography was then taken to confirm the fracture. After the surgery, the 30 mice were randomly assigned into one of three groups: the Luc-MSC local injection group (Loc group), the Luc-MSC systemic injection group (Sys group), or the PBS control group (PBS group); 10 mice were in each group.

\section{Cell injection}

In this study, we used cardiac injection of MSCs directed by an ultrasound imaging system (Vevo 770; VisualSonics Inc., Toronto, ON, Canada) instead of intravenous injection, based on the following reasons: (1) the blood vessels in the mouse tail vein are thin; therefore, it is hard to be penetrated by the needle; moreover, the needle may break the vessel, resulting in cell leaking. (2) Our preliminary study showed that the cells injected through either the tail vein or the heart all ravel to the lungs within minutes of injection. There are more cells trapped in the lungs in the vein injection group comparing to the heart injection group.

Cells and PBS injection were carried out at 4 days after fracture. For the Luc-MSC systemic injection group, $5 \times 10^{5}$ Luc-MSCs (in $100 \mu$ l of PBS) were injected into the left ventricle through heart puncture under the ultrasound imaging system; mice in the PBS control group were given $100 \mu \mathrm{l}$ of PBS injection through heart puncture as above; for the local injection group, $5 \times 10^{5}$ Luc-MSCs (in $100 \mu \mathrm{l}$ of PBS) were directly injected into the fracture site.

\section{In vivo bioluminescent assays}

After cell injection, five mice per Loc and Sys group were intra-peritoneally injected with D-Luciferin $(15 \mathrm{mg} / \mathrm{ml}, 300 \mu \mathrm{l}$ for a 30-g mouse). After 10 minutes, mice were subjected to the IVIS imaging examination, and the region of interest (ROI) was set in each image. The same parameter settings for IVIS imaging were used for all samples in this study: $f$ number: 1 , field of view: 22, binning factor: 16, luminescent exposure (seconds): 10. Mice were examined by IVIS imaging system every 2 days and thereafter until the signal disappeared. The rate of photons per second of ROI was calculated by IVIS software, the data were then analyzed by SPSS statistical software, and the intensity of the signal was expressed as percentages of photons per second of ROI. To minimize the variations between individuals for IVIS imaging detection, the IVIS 200 system was calibrated each day for the first use in accordance with the manufacturer's manual, and all the IVIS in vivo imaging detection was carried out by two investigators who were blinded to the groups of animals tested (with animal coding unknown to the IVIS imaging machine operator).

\section{Micro-computer tomography examination}

All 30 mice were terminated at 5 weeks after fracture. Eight mice per group were randomly chosen for microCT analysis. Right femurs of all 30 mice were excised; muscles, soft tissues, and the internal stainless pins were carefully removed. For image acquisition, 300 two-dimensional (2D) micro-tomographic slices with a $20-\mu \mathrm{m}$ slice increment covering a total range of $6 \mathrm{~mm}$ were scanned by Scanco Medical $\mu$ CT40 (Scanco Ltd., Brüttisellen, Switzerland). Two hundred fifty sequential slices of 2D CT images at $2.5 \mathrm{~mm}$ proximal and $2.5 \mathrm{~mm}$ distal to the fracture line were selected, and the contoured region including the cortical diaphyseal bone and endosteal callus was set in each image. A low-pass Gaussian filter (Gauss sigma $=0.8$ and Gauss support $=1$ ) was used to partly suppress the noise in the volumes. The high- and low-radio-opacity mineralized tissues were segmented by thresholding, and an appropriate threshold was determined from the grayscale CT images. Bone volume (BV), tissue volume (TV), BV/TV, and mean volumetric bone mineral density for each sample were recorded.

\section{Three-point bending mechanical testing}

Tests were performed within 24 hours after excision at room temperature, and fractured femurs were tested to failure with a constant displacement rate of $4 \mathrm{~mm} /$ minute by a three-point bending device (H25KS; Hounsfield Test Equipment Ltd., Redhill, Surrey, UK). The femurs were loaded in the anterior-posterior direction with the span of the two support points set as $8 \mathrm{~mm}$. The force loading point was set at the fracture site. After testing, the loaddisplacement curves of the femurs were generated by the built-in software (QMAT Professional Material testing software; Hounsfield Test Equipment Ltd.); ultimate load to failure, energy absorbed to failure (the area under the load-displacement curves, known as the toughness), and the modulus of elasticity (E-Modulus, the slope of the stress-strain curve, known as the tissue stiffness) [30] were recorded and analyzed by the software.

\section{Histology and immunohistochemistry}

The fractured femurs were fixed in $4 \%$ buffered formalin for 1 day and then decalcified with $9 \%$ formic acid for 5-7 days. Attempts were made to standardize the sectioning at a mid-sagittal plane of each specimen by cutting the specimen in half (longitudinally in a sagittal plane) by using a slicing blade. Samples were subjected for tissue processing and then embedded in paraffin. Thin sections $(5 \mu \mathrm{m})$ were cut on a Rotary Microtome (HM 355S; Thermo Fisher Scientific, Dreieich, Germany) along the long axis of each 

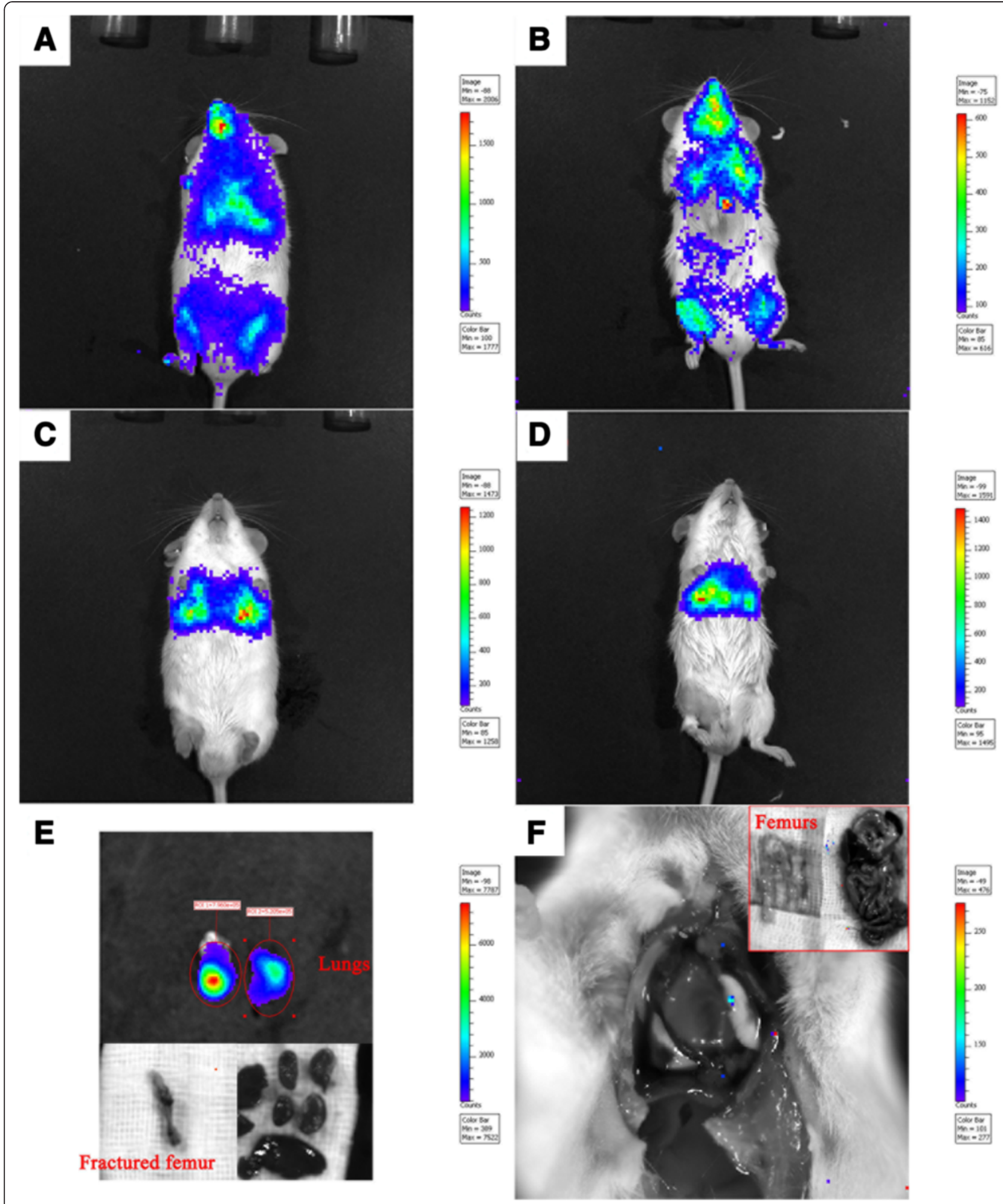

$\mathbf{E}$

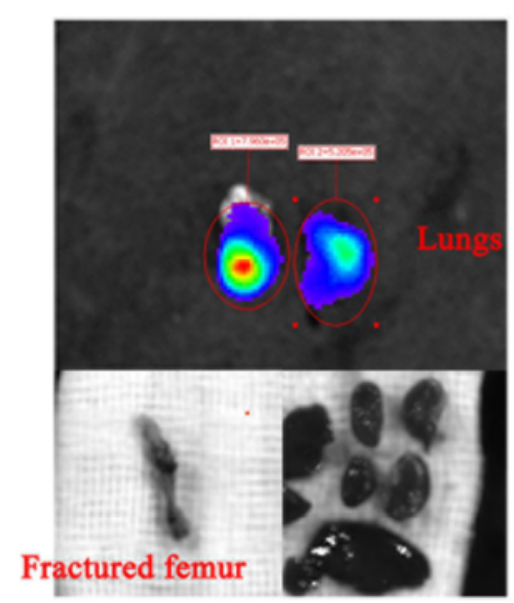

Fig. 1 In vivo tracking systemically injected Luc-MSCs. a, b During the first hour following the injection, Luc-MSCs spread throughout the whole body via circulating system, and high concentrations of Luc-MSCs were observed in limbs, lungs, and oral cavity. c, d After about 1 hour following the injection, high concentrations of Luc-MSCs were observed only at the lung site, but no signal was observed at limbs, the oral cavity, and other parts of the body. e At day 4 following the injection, one mouse was terminated, and strong signals were observed at excised lungs, but no signal was observed on the excised fractured femur and other organs. f At day 11 following the injection, another mouse was terminated; no signal was observed throughout the whole body, including the lung site. LuC-MSC Luciferase labeled mesenchymal stem cell 
femur in the sagittal plane. Sections were mounted on the coated slides. Paraffin was removed by immersing the slides in Xylene 2 changes of 5 minutes at room temperature. Slides were then taken through graded ethanol and distilled water. For antigen retrieval, slides were immersed in $10 \mathrm{mM}$ of citrate buffer at $60{ }^{\circ} \mathrm{C}$ for 20 minutes and then rinsed with PBS twice; the slides were then immersed in $3.0 \%$ hydrogen peroxide in PBS for 5 minutes and rinsed twice with PBS for 5 minutes each.

\section{TUNEL assay and immunofluorescence staining}

A peroxidase in situ apoptosis detection kit (catalog number S7100; EMD Millipore, Billerica, MA, USA) was used to detect the apoptosis level of injected LucMSCs. In brief, following the step above, slides were taken through equilibration buffer for 10 seconds and TDT enzyme in a humidified chamber at $37{ }^{\circ} \mathrm{C}$ for 1 hour. The slides were washed in PBS buffer for 10 minutes, then anti-digoxignenin conjugate was applied on the slides for 30 minutes, and this was followed by PBS washing and peroxidase substrate incubation for 6 minutes. The slides were finally counterstained in $0.5 \%$ (wt/vol) methyl green for 10 minutes and washed in PBS. For detection of Luc-MSCs on the same slide, we performed immunostaining. Briefly, the counterstained slides were blocked by $5 \%$ donkey serum in $1 \%$ bovine serum albumin (BSA) for 20 minutes and then incubated with the goat anti-Luciferase antibody (1:300; Santa Cruz Biotechnology, Inc., Dallas, TX, USA) overnight at $4{ }^{\circ} \mathrm{C}$. Slides were washed with PBS three times, incubated with the donkey anti-goat IgGFITC (1:1000; Santa Cruz Biotechnology, Inc.) for 60 minutes at room temperature in the dark, and washed and mounted. The fluorescent cells were visualized by using a fluorescent microscope (Zeiss-spot; Carl Zeiss MicroImaging GmbH, Jena, Thuringia, Germany).

\section{Double immunofluorescence staining}

We used two antibodies in one slide. In brief, slides were blocked by $5 \%$ donkey serum in $1 \%$ BSA for 20 minutes, incubated with the goat anti-Luciferase antibody (1:300; Santa Cruz Biotechnology, Inc.) and the rabbit antiOsteocalcin antibody (1:300; Santa Cruz Biotechnology, Inc.) or the rabbit anti-Nestin antibody (1:300; SigmaAldrich) overnight at $4{ }^{\circ} \mathrm{C}$, washed with PBS three times, and incubated with the donkey anti-goat IgG-FITC (1:1000; Santa Cruz Biotechnology, Inc.) and Cy3 donkey anti-rabbit IgG (1:1000; Life Technologies, Carlsbad, CA, USA) for 60 minutes at room temperature in the dark.

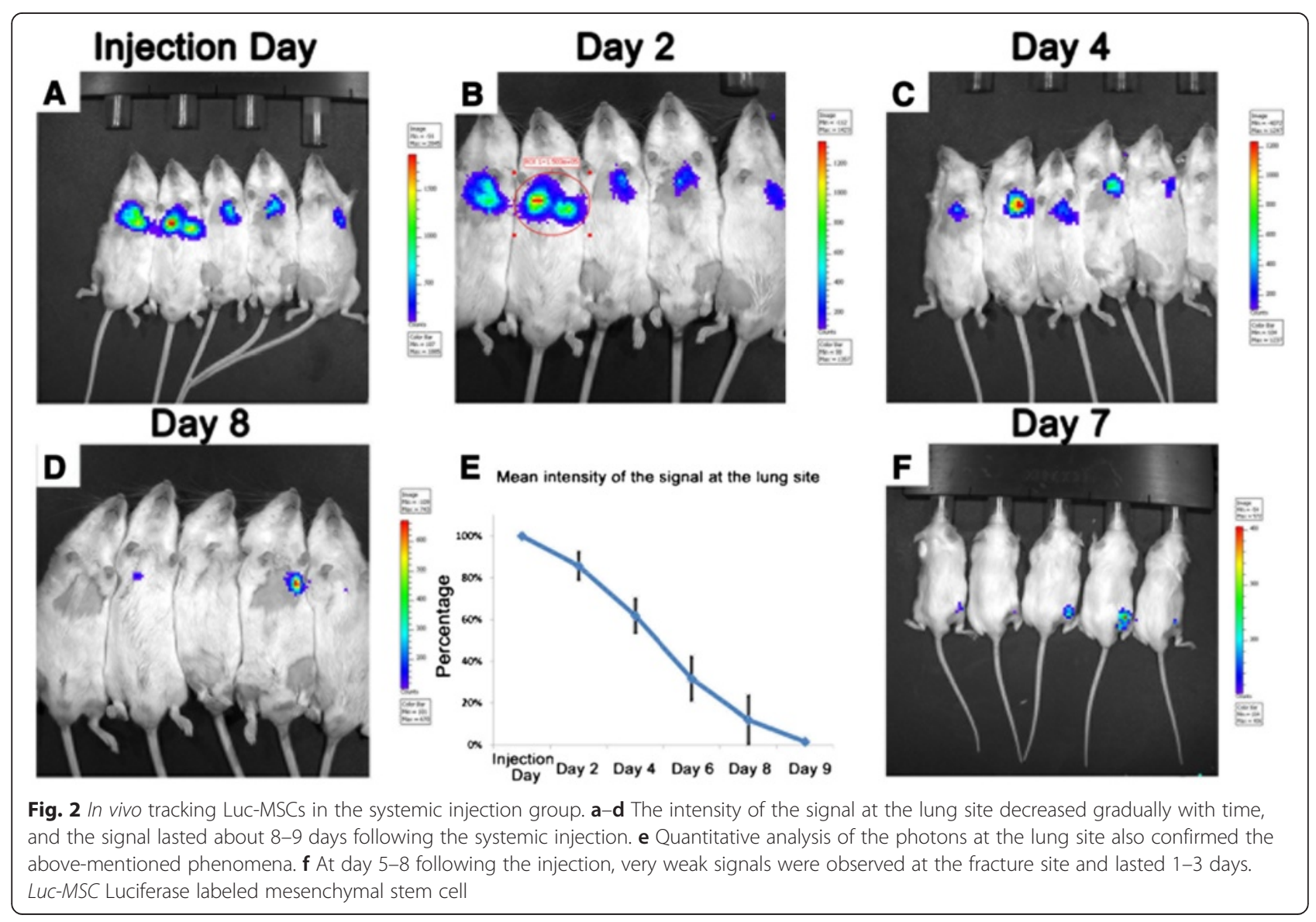


Slides were washed with PBS three times, counterstained with 4',6-diamidino-2-phenylindole (DAPI), mounted, and examined under fluorescent microscope.

\section{Quantitation of injected Luc-positive cells at the fracture site}

The image with the maximum callus width in each sample was chosen for cell counting. Briefly, the image was opened by ImageJ version 1.48 (National Institute of Mental Health, Bethesda, MD, USA); when "Plugins/ Analyze/Cell Counter" plugin is run, the Crosshair (mark and count) tool was used to manually count apoptotic and immunofluorescence-labeled cells. The cell counting result was generated by the software.

\section{Statistical analysis}

All quantitative data were transferred to statistical spreadsheets and analyzed by a commercially available statistical program: SPSS version 16.0 (IBM Corporation, Armonk, NY, USA). One-way analysis of variance followed by post hoc test was used for comparison of mean values, and $P$ values of less than 0.05 were considered statistically significant.

\section{Results}

The characterization of MSCs

The flow cytometry results confirmed that isolated LucMSCs were negative for hematopoietic marker CD45 (Additional file 1: Figure S1A) and endothelial cell marker CD31 (Additional file 1: Figure S1B) and homogenously positive for MSC markers CD44 and CD90 (Additional file 1: Figure S1C, D). In differentiation assays of Luc-MSCs, the Alizarin red staining demonstrated that mineralized nodules formed after 3 weeks of the osteogenic induction (Additional file 1: Figure S1E); intracellular Oil red O-stained lipid-rich vacuoles appeared after 2 weeks of the adipogenic induction (Additional file 1: Figure S1F).

\section{In vivo bioluminescent assays}

In the systemic injection group at the first hour following injection, Luc-MSCs spread throughout the body via circulating system, and higher concentrations of Luc-MSCs were seen in limbs, lungs, and oral cavity (Fig. 1a, b). After 1 hour following the injection, the Luc-MSCs aggregated in the lungs, and the signal at limbs, oral cavity, and other locations of the body was diminished (Fig. 1c, d). At day 4 following the injection, one mouse was terminated; the lungs, femurs, heart, liver, and kidney were excised and

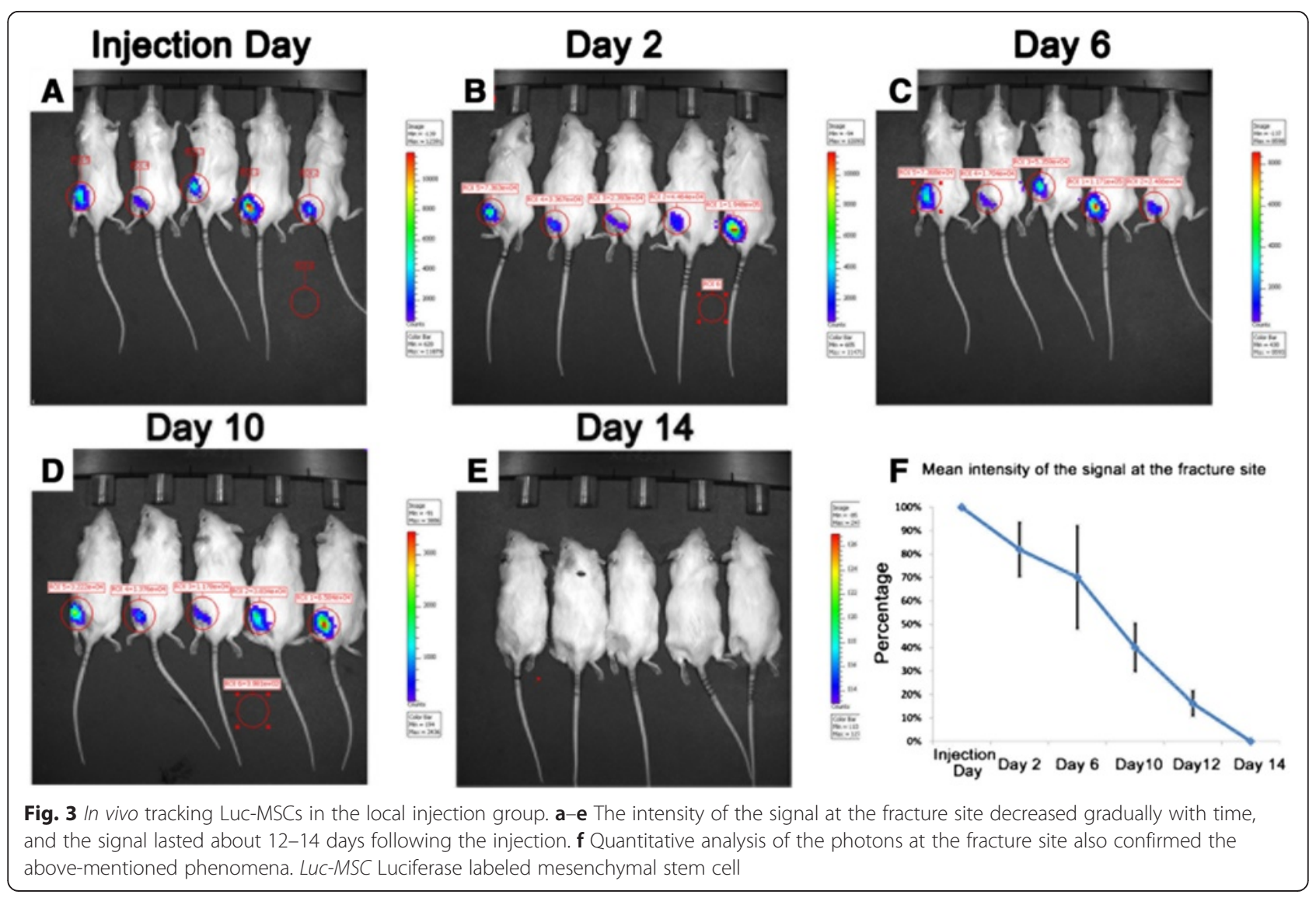


scanned by using the IVIS system; a strong signal was seen in the lungs, but none was seen at the fractured femur and other organs (Fig. 1e). At day 11 following the injection, another mouse was terminated, and there was no signal throughout the whole body, including the lung site (Fig. 1f). Figure 2a-d showed that the intensity of the signal at the lung site decreased gradually with time, and the detectable signals lasted about 8-9 days following the injection. Quantitative comparison of photons at different time points confirmed the continuous disappearance of the Luc-MSCs in the lungs (Fig. 2e). At day 5-8 following the injection, weak signals appeared at the fracture site (Fig. 2f) and lasted 1-3 days and then disappeared.

In the local injection group, the intensity of the signal at the fracture site decreased gradually with time, and the signal lasted about 12-14 days following the injection (Fig. 3a, b, c, d, e) and that was confirmed by quantitative comparison of photons at different time points (Fig. 3f).

\section{Micro-CT analysis of the fractured bone}

Quantitative analysis revealed that the mean density of bone volume in the PBS control group was significantly lower than that of the MSC systemic injection group $(P<0.05)$ and the MSC local injection group $(P<0.05)$, but there was no significant difference between the MSC systemic injection group and the MSC local injection group (Fig. 4a). The bone volume over tissue volume (BV/TV) in the MSC local injection group was significantly higher than that of the PBS control group $(P<0.05)$, but there was no significant difference between the MSC systemic injection group and the MSC local injection group (Fig. 4b).

\section{Three-point bending mechanical testing}

E-Modulus (known as the tissue stiffness) in the MSC local injection group was significantly higher than that of the PBS control group $(P<0.05)$, but there was no significant difference between the MSC systemic injection group and the MSC local injection group $(P>0.05)$ and also no significant difference between the PBS control and the MSC systemic injection group $(P>0.05)$ (Fig. 4c).

Ultimate load to failure in the MSC systemic injection group was significantly higher than that of the PBS control group $(P<0.05)$, and the MSC local injection group was significantly higher than that of the PBS control group $(P<0.05)$, but there was no significant difference between the MSC systemic injection group and the MSC local injection group $(P>0.05)$ (Fig. 4d). Energy absorbed to failure (known as the toughness) did not

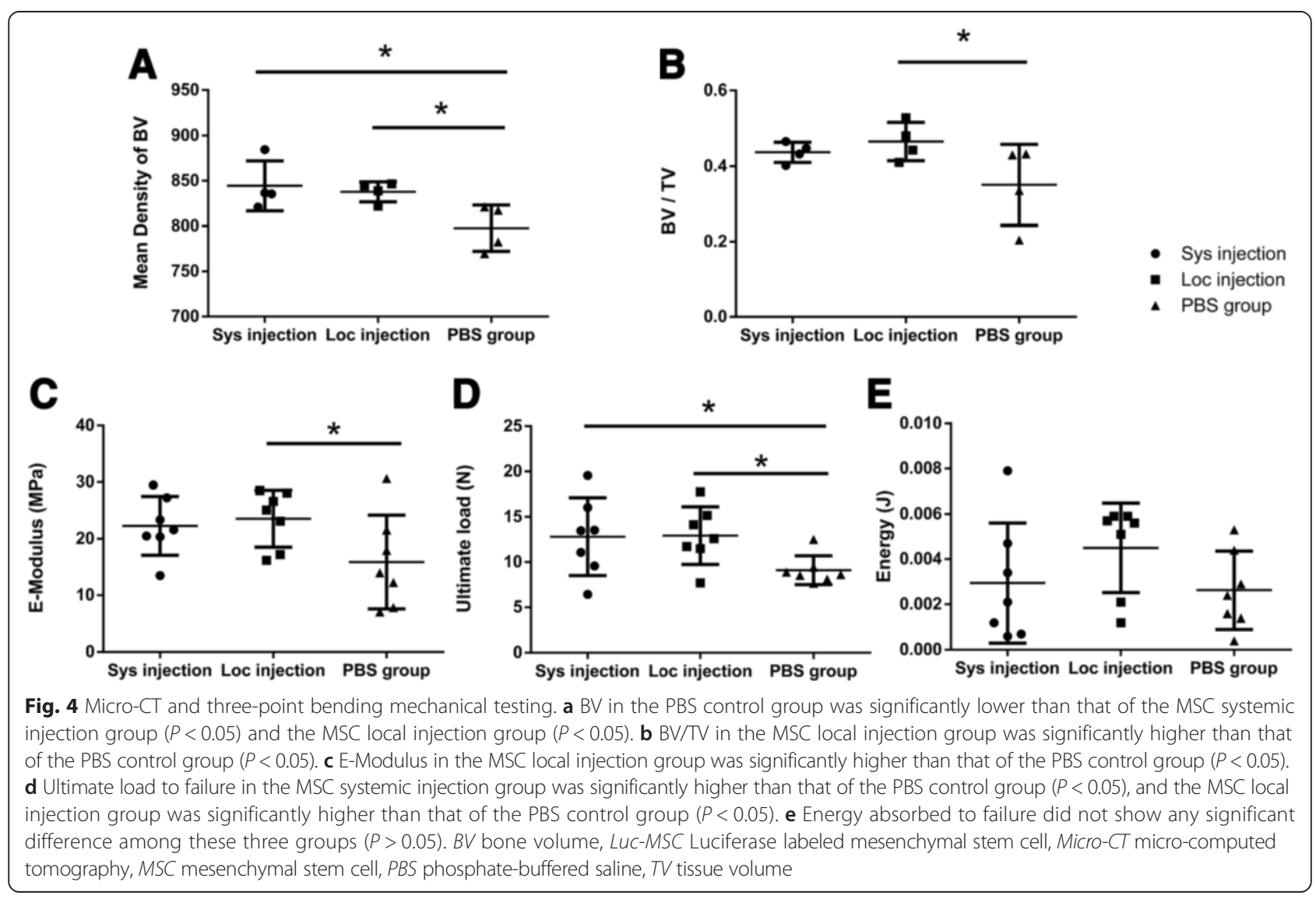


show any significant difference among these three groups $(P>0.05)$ (Fig. 4e).

\section{Detection of apoptotic Luc-MSCs}

To examine the apoptosis level of injected Luc-MSCs, peroxidase staining and immunofluorescence staining were carried out on the same section. A mass of apoptotic cells (brown cells) was observed around the fracture sites within the callus in the Luc-MSC systemic injection group (Fig. 5a), the local injection group (Fig. 5b), and the PBS control group (Fig. 5c) at 5 weeks following the fracture, but none was observed in the negative control (the normal femur) (Fig. 5d).

High-resolution images were respectively enlarged from the red outlined areas in Fig. 5a, b, c, d (Fig. 5e, f, g, h). Both living Luc-positive cells (light green cells within red circles) and apoptotic Luc-positive cells (dark green cells within white circles) were found in the MSC systemic injection group (Fig. 5e) and the MSC local injection group (Fig. 5f) at 5 weeks following the fracture, but no
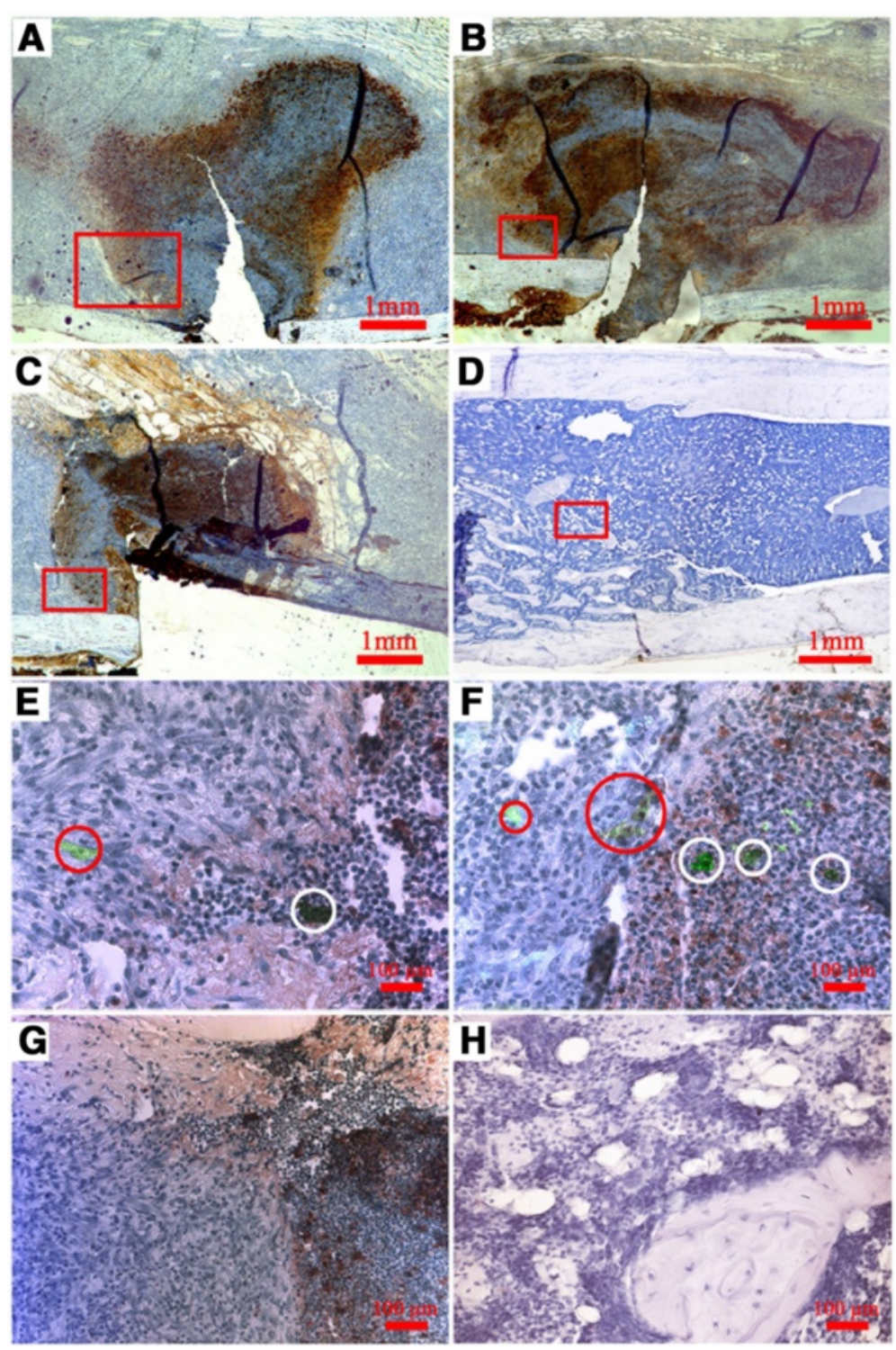

Fig. 5 Illustration of apoptotic cells at the fracture site. A mass of apoptotic cells (brown cells) was observed around the fracture ends within the callus in the Luc-MSC systemic injection group (a), the local injection group (b), and the PBS control group (c) at 5 weeks following the fracture, but none was observed in the negative control (the normal femur) (d). e-h High-resolution images were, respectively, enlarged from the red outlined areas in a-d. Both living Luc-positive cells (light green cells within red circles) and apoptotic Luc-positive cells (dark green cells within white circles) were found in the MSC systemic injection group (e) and the MSC local injection group (f) at 5 weeks following the fracture, but no Luc-positive cells were found in the PBS control group $(\mathbf{g})$ and the negative control (h). LuC-MSC Luciferase labeled mesenchymal stem cell, MSC mesenchymal stem cell, PBS phosphate-buffered saline 
Luc-positive cells were found in the PBS control group (Fig. 5g) and the negative control (Fig. 5h).

\section{Double immunofluorescence staining for Luc/Nestin and Luc/Osteocalcin}

To examine the co-distribution of luciferase (FITC immunolabeling) and Nestin (Cy3 immunolabeling), an MSC marker, double immunofluorescence staining was carried out on the same section. $\mathrm{Luc}^{+} / \mathrm{Nestin}^{+}$cells, the redyellow cells pointed out by red arrows in Fig. 6a, were observed in the MSC systemic injection group, which meant that some systemic injected allogenic Luc-MSCs had migrated to the fracture site via circulation system and still maintain their phenotype at 5 weeks following
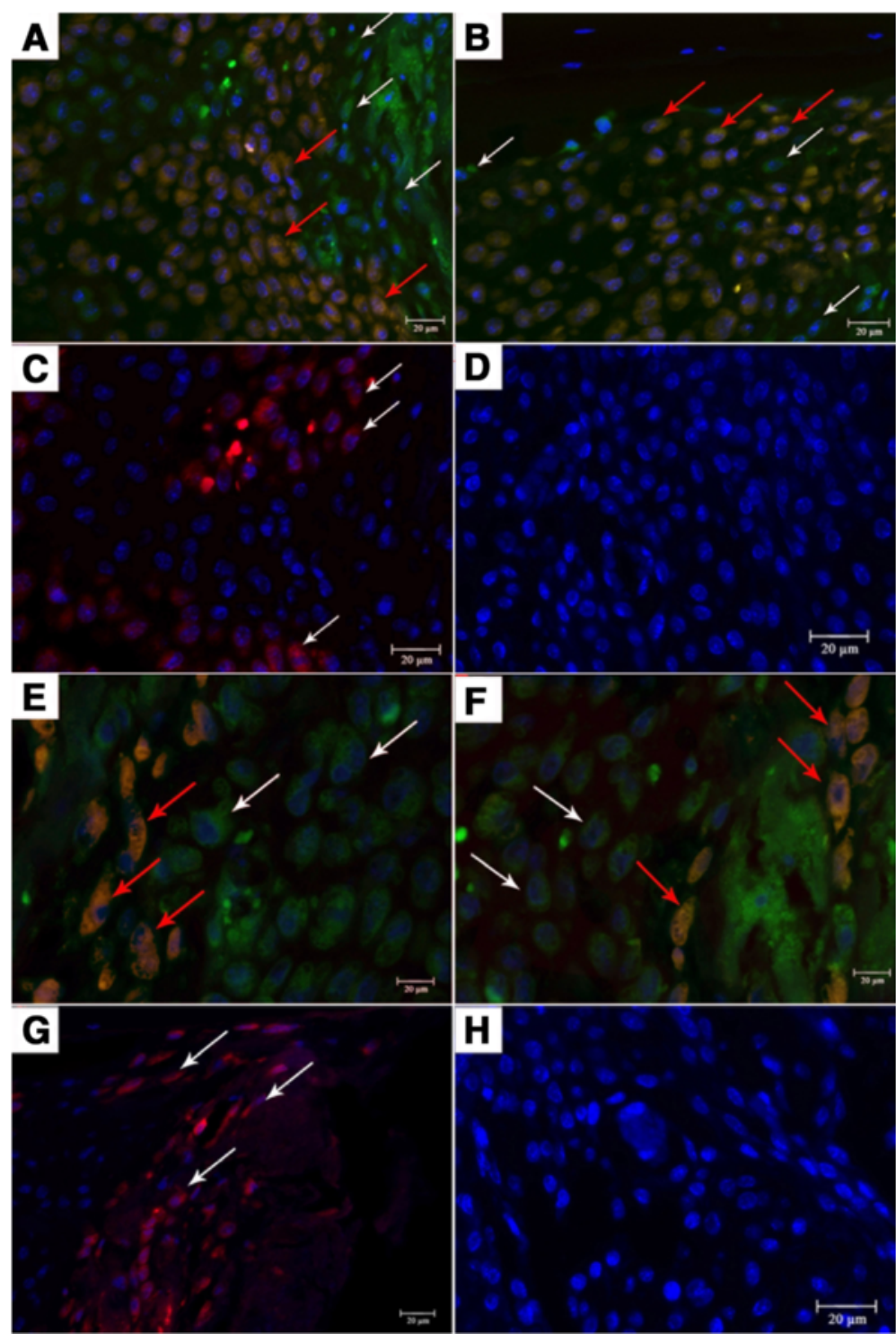

Fig. 6 Double immunofluorescence staining for Luc/Nestin and Luc/Osteocalcin (DAPI for nucleuses). a In the Sys group, Luc ${ }^{+} /$Nestin $^{+}$cells (the red-yellow cells pointed out by red arrows), the systemically injected allogenic Luc-MSCs, had migrated to the fracture site. $\mathbf{b}$ In the Loc group, Luc $^{+} /$Nestin $^{+}$cells (red arrows), the injected Luc-MSCs through local sites, remained at 5 weeks following the fracture. Luc ${ }^{+} / \mathrm{Nestin}^{-}$cells (the green cells pointed out by white arrows in a, b) were observed, which meant that some injected Luc-MSCs had differentiated to other types of cells in both MSC injection groups. c, d Luciferase-positive cells were not observed in the PBS group (c) and the negative control (d), but Luc ${ }^{-}$/Nestin ${ }^{+}$cells (white arrows in c), autogenetic MSCs, existed in the callus in the PBS control group. e, $\mathbf{f}$ Luc $^{+} / \mathrm{Osteocalcin}^{+}$cells (the red-yellow cells pointed out by red arrows) were observed both in the Sys group (e) and the Loc group (f), which meant that some injected Luc-MSCs had differentiated to osteoblasts. Luc ${ }^{+} /$Osteocalcin ${ }^{-}$cells, the green cells (white arrows), were also found in MSC injection groups, which meant that some injected Luc cells remained at 5 weeks following the fracture. $\mathbf{g}, \mathbf{h}$ Luciferase-positive cells were not observed in the PBS control group ( $\mathbf{g}$ ) and the negative control (h), but Luc ${ }^{-}$Osteocalcin ${ }^{+}$cells, autogenetic osteoblasts, were found in the callus. Scale bar: 20 Mm. DAPI 4,6-diamidino2-phenylindole, Luc-MSC Luciferase labeled mesenchymal stem cell, MSC mesenchymal stem cell, PBS phosphate-buffered saline 
the fracture. $\mathrm{Luc}^{+} / \mathrm{Nestin}^{+}$cells (red arrows, Fig. 6b) were observed in the MSC local injection group, which meant that some injected Luc-MSCs through local sites remained at 5 weeks following the fracture. $\mathrm{Luc}^{+} / \mathrm{Nestin}^{-}$ cells, the green cells pointed out by white arrows in Fig. $6 \mathrm{a}$, b, were also observed, which meant that some injected Luc-MSCs had differentiated into other types of cells in both MSC injection groups. Luciferase-positive cells were not observed in the PBS control group (Fig. 6c) and the negative control (without the primary antibodies) (Fig. 6d), but $\mathrm{Luc}^{-} / \mathrm{Nestin}^{+}$cells were found in the PBS control group (white arrows in Fig. 6c), which meant that autogenetic MSCs existed at the fracture site in the PBS control group.

Double immunofluorescence staining was also carried out to examine the co-distribution of luciferase (FITC immunolabeling) and Osteocalcin (Cy3 immunolabeling), secreted solely by osteoblasts on the same section. $\mathrm{Luc}^{+} / \mathrm{Osteocalcin}^{+}$cells, the red-yellow cells pointed out by red arrows, were observed in both the MSC systemic injection group (Fig. 6e) and the MSC local injection group (Fig. 6f) at 5 weeks following the fracture, which meant that some injected Luc-MSCs had differentiated into osteoblasts at 5 weeks following the fracture and contributed to the bone formation. Luc $^{+} /$Osteocalcin ${ }^{-}$ cells, the green cells pointed out by white arrows in Fig. 6e, f, were also found in both systemic and local injection groups, which confirmed that some injected Luc-positive cells remained at the fracture sites at 5 weeks following the fracture. Luc-positive cells were not observed in the PBS control group (Fig. 6g) and the negative control (without primary antibodies) (Fig. 6h), but Luc -/Osteocalcin ${ }^{+}$cells were found in the PBS control group (white arrows in Fig. 6g), which meant that autogenetic osteoblasts existed at the fracture site at 5 weeks following the fracture and contributed to the bone formation.

\section{The number of injected Luc-positive cells at the fracture} site

Quantitative analysis revealed that the number of Lucpositive cells in the callus in the MSC systemic injection group was significantly lower than that of the MSC local injection group $(P<0.05)$ (Fig. 7a). The proportion of apoptotic Luc-positive cells in Luc-positive cells in the

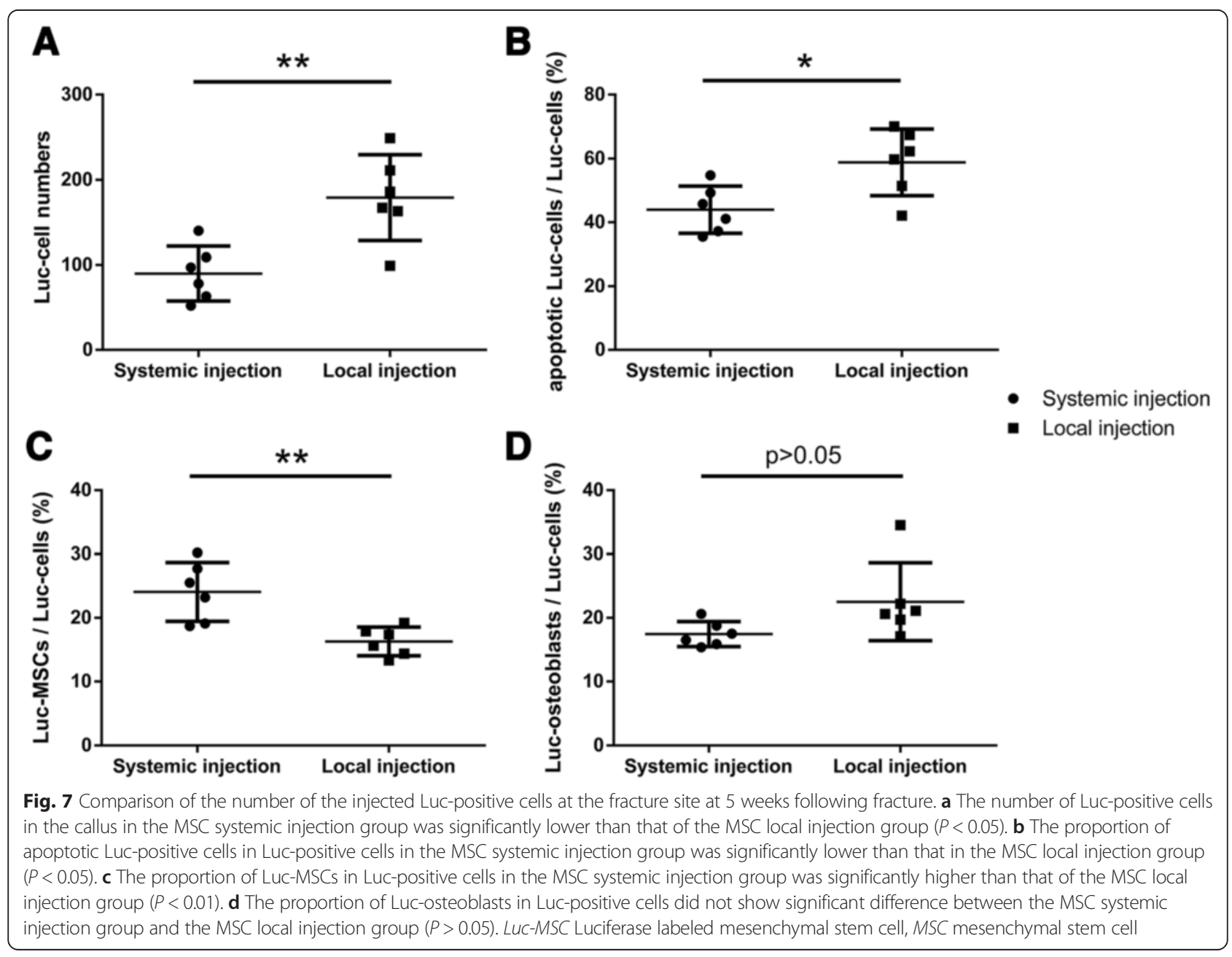


MSC systemic injection group was significantly lower than that in the MSC local injection group $(P<0.05)$ (Fig. 7b). The proportion of Luc-MSCs in Luc-positive cells in the MSC systemic injection group was significantly higher than that of the MSC local injection group $(P<0.01)$ (Fig. 7c). The proportion of Luc-osteoblasts in Luc-positive cells did not show a significant difference between the MSC systemic injection group and the MSC local injection group $(P>0.05)$ (Fig. $7 d)$.

\section{The minimal Luc cells that IVIS in vivo imaging system could detect in vivo in mice}

IVIS in vivo imaging systems, based on the principle of bioluminescence, are widely used for in vivo tracking and monitoring of Luciferase-expressing cells in recipient animals [29, 31]. However, it was reported that Luciferase expression was observed to decrease with time, most likely the result of tissue rejection [32]. The light signal detected on the surface of the mouse also depends on the depth of the injected cells below the tissue surface and the number of Luc cells. To test the sensitivity and the accuracy of the IVIS imaging system, we subcutaneously injected $1.5 \times 10^{4}, 3 \times 10^{4}$, and $4.5 \times 10^{4}$ Luc-MSCs with luciferin, respectively, into three different areas of the nude mouse and the FVB mouse (with hair) (area I: $4.5 \times 10^{4}$ cells, II: $3 \times 10^{4}$ cells, III: $1.5 \times 10^{4}$ cells; Fig. 8), and then we monitored both mice by using IVIS. The results showed that area III in the FVB mouse did not show any signal under IVIS but did in the nude mouse (Fig. 8). Concerning the foregoing results, in the systemic injection group, the signal at the fracture site was found only at day 5-8 following the injection by IVIS; in the local injection group, the signal at the fracture site lasted only 12-14 days; however, Luc-positive cells could be found by immunofluorescence staining at 5 weeks following the fracture in both MSC injection groups; therefore, in the present studies, we concluded that the IVIS 200 in vivo imaging system was sensitive to detect the number of the Luc cells above $1.5 \times 10^{4}$ in vivo in mice.

\section{Discussion}

\section{Systemic and local injection of allogenic MSCs promoted} fracture healing equally in this study

Systemic injection is more appropriate for treatments of systemic diseases such as osteoporosis, injuries involving deeper sites, and poly-trauma; and local injection is simpler for single and superficial injures such as skin wound or burns [33]. The present study has demonstrated that both systemic and local administration of allogeneic MSCs promoted fracture repair significantly, and obvious immune responses in this study were not observed in either allogeneic MSC systemic or local administration. Therefore, the use of allogeneic MSCs either systemically or locally is effective in the mouse fracture model.

The explanations for the equal effects in promoting fracture healing in both systemic and local injection groups are that (1) the limited sample size used in this

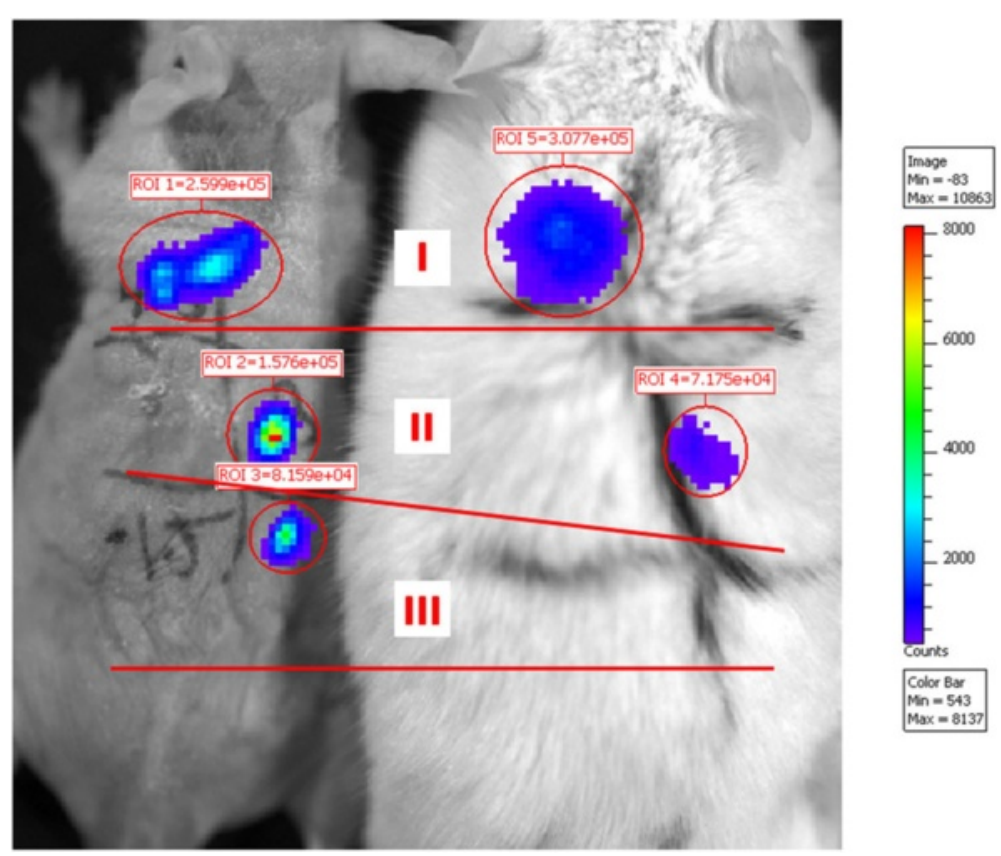

Fig. 8 In vivo tracking injected Luc-positive cells by IVIS 200. Luc-MSCS $\left(1.5 \times 10^{4}, 3 \times 10^{4}\right.$, and $\left.4.5 \times 10^{4}\right)$ with luciferin were subcutaneously injected into three different areas of the nude and FVB (with hairs) mouse (area l: $4.5 \times 10^{4}$ cells, II: $3 \times 10^{4}$ cells, III: $1.5 \times 10^{4}$ cells). Luc cells $\left(1.5 \times 10^{4}\right)$ in the FVB mouse cannot be found by IVIS 200. Luc-MSC Luciferase labeled mesenchymal stem cell, MSC mesenchymal stem cell 
study may not verify the small difference, (2) systemically and locally injected cells may contribute to the fracture healing in different ways, (3) high dose of injected cells ensured the equal effects, and (4) our data showed that the number of systemically injected Luc cells at the fracture site were significantly less than those of locally injected Luc cells because of the blood barriers in the lungs; however, the proportion of apoptotic Luc-positive cells in Luc-positive cells in the MSC systemic injection group was also significantly lower than that in the MSC local injection group, which may be credited to the low $\mathrm{pH}$, hypoxia, inflammation, and infection in the local environment.

The in vivo imaging is a very useful technique and an invaluable tool to examine the living cells in vivo, and at present the IVIS system is the best research tool for tracing luciferase-labeled cells in vivo in small animals (mice). However, there is a limitation of IVIS system, and in the present study we found that the IVIS 200 system was not sensitive enough to detect the number of the Luc cells below $1.5 \times 10^{4}$ in vivo in mice; hence, the use of immunohistochemistry methods to detect the Luc-positive cells will be necessary to confirm the whereabouts of the Luc-positive cells when the cells numbers are expected to be lower.

\section{The fate of engrafted MSCs}

In vivo imaging data showed the following: (1) For heart injection, MSCs spread throughout the whole body via the circulating system in the early hours after the injection and aggregated into lungs, gradually decreased with time, and disappeared after about 8-9 days. Injected MSCs could be found at the fracture site at day 5-8 following the injection. (2) For local injection, engrafted MSCs at the fracture site decreased gradually with time, and the Luc cells disappeared at about 12-14 days following the injection.

Our double Immunofluorescence staining results showed that both systemically and locally injected LucMSCs were observed in the callus even at 5 weeks following the fracture, and some of them had differentiated into osteoblasts and directly contribute to the bone formation. However, as we discussed above, IVIS 200 was not sensitive to the number of the Luc cells below $1.5 \times 10^{4}$ in vivo in the FVB mouse, which meant that less than $3 \%$ of injected Luc cells $\left(1.5 \times 10^{4} / 5 \times 10^{5}\right)$ remained at the fracture site in the MSC injection groups at 5 weeks following the fracture, and about half of these cells went apoptosis in the callus. Our preliminary studies also showed that the systemically injected Luc-MSCs were not found in the heart, liver, kidney, and lungs by using immunofluorescence staining at 5 weeks following the fracture, which meant that almost $98.5 \%$ of the injected Luc cells were dead after 1 month following the transplantation.

\section{Conclusions}

In this study, we tracked and estimated the systemically and locally injected MSCs in both lungs and fracture sites by using in vivo imaging system, which may give guidance for the clinical transplantation of MSCs. Our data also showed that both systemic and local administration of allogeneic bone marrow-derived MSCs promoted mouse fracture healing and enhanced callus formation and their mechanical properties. However, we have not understood the exact cellular and molecular mechanisms behind the systemic allogeneic MSC administration in promoting fracture repair, and they still need further investigations.

\section{Additional file}

Additional file 1: Figure S1. Cell surface markers and differentiation capacities of mouse MSCs. (A) Flow cytometry analysis results confirmed that isolated BM-MSCs were negative for hematopoietic marker CD45. (B) Cells were negative for endothelial cell marker CD31. (C, D) Cells were positive for MSC markers CD44 and CD90. (E) The Alizarin red staining demonstrated that mineralized nodules formed after 4 weeks of the osteogenic induction. (F) Intracellular Oil red O-stained lipid-rich vacuoles appeared after 2 weeks of the adipogenic induction. BM-MSC bone marrow-derived mesenchymal stem cell, MSC mesenchymal stem cell. (TIFF $468 \mathrm{~kb}$ )

\section{Abbreviations}

BSA: Bovine serum albumin; BV: Bone volume; FITC: Fluorescein isothiocyanate; Luc-MSC: Luciferase labeled mesenchymal stem cell; MicroCT: Micro-computed tomography; MSC: Mesenchymal stem cell; PBS: Phosphate-buffered saline; TV: Tissue volume.

\section{Competing interests}

The authors declare that they have no competing interests.

\section{Authors' contributions}

SH carried out the cell culture, all animal experiments, and data analysis. YS and $Y Z$ participated in the animal experiments, data collection, and analysis. LX participated in the design of the study and performed the data collection and statistical analysis. GL conceived of the study and participated in its design and coordination. All authors have been involved in drafting the manuscript or revising it critically and approved the final manuscript.

\section{Acknowledgments}

This work is supported by a grant from Hong Kong Government Research Grant Council, General Research Fund (CUHK470813), and a grant from China Shenzhen City Science and Technology Bureau under the Shenzhen City Knowledge Innovation Plan, Basic Research Project (JCYJ20130401171935811) to Gang Li. This study was also supported in part by SMART program, Lui Che Woo Institute of Innovative Medicine, Faculty of Medicine, the Chinese University of Hong Kong. This research project was made possible by resources donated by Lui Che Woo Foundation Limited.

\section{Author details}

'Department of Orthopaedics \& Traumatology, Faculty of Medicine, Room 904, 9/F, Li Ka Shing Institute of Health Institute, Prince of Wales Hospital, The Chinese University of Hong Kong, Shatin, 30-32 Ngan Shing Street, Shatin, NT, Hong Kong, SAR, PR China. ${ }^{2}$ Stem Cells and Regenerative Medicine Laboratory, Li Ka Shing Institute of Health Sciences, The Chinese University of Hong Kong, Prince of Wales Hospital, 30-32 Ngan Shing Street, 
Shatin, NT, Hong Kong, PR China. ${ }^{3}$ Key Laboratory for Regenerative Medicine, Ministry of Education, School of Biomedical Sciences, Faculty of Medicine, The Chinese University of Hong Kong, Shatin NT, Hong Kong, SAR, China. ${ }^{4}$ The CUHK-ACC Space Medicine Centre on Health Maintenance of Musculoskeletal System, The Chinese University of Hong Kong Shenzhen Research Institute, No. 10, 2nd Yuexing Road, South District, Hi-tech Park, Nanshan, 518057 Shenzhen, PR China. ${ }^{5}$ Lui Che Woo Institute of Innovative Medicine, Faculty of Medicine, The Chinese University of Hong Kong, 30-32 Ngan Shing Street, Shatin, NT, Hong Kong, SAR, China.

Received: 7 March 2015 Revised: 23 July 2015

Accepted: 5 October 2015 Published online: 26 October 2015

\section{References}

1. Pittenger MF, Mackay AM, Beck SC, Jaiswal RK, Douglas R, Mosca JD, et al. Multilineage potential of adult human mesenchymal stem cells. Science. 1999;284:143-7.

2. Sanchez-Ramos JR. Neural cells derived from adult bone marrow and umbilical cord blood. J Neurosci Res. 2002;69:880-93.

3. Pereira RF, O'Hara MD, Laptev AV, Halford KW, Pollard MD, Class R, et al. Marrow stromal cells as a source of progenitor cells for nonhematopoietic tissues in transgenic mice with a phenotype of osteogenesis imperfecta. Proc Natl Acad Sci U S A. 1998;5:1142-7.

4. Lee RH, Seo MJ, Reger RL, Spees JL, Pulin AA, Olson SD, et al. Multipotent stromal cells from human marrow home to and promote repair of pancreatic islets and renal glomeruli in diabetic NOD/scid mice. Proc Natl Acad Sci U S A. 2006;103:17438-43.

5. Le Blanc K, Rasmusson I, Sundberg B, Gotherstrom C, Hassan M, Uzunel M, et al. Treatment of severe acute graft-versus-host disease with third party haploidentical mesenchymal stem cells. Lancet. 2004;363:1439-41.

6. Li H, Guo Z, Jiang X, Zhu H, Li X, Mao N. Mesenchymal stem cells alter migratory property of $T$ and dendritic cells to delay the development of murine lethal acute graft-versus-host disease. Stem Cells. 2008;26:2531-41.

7. Bruder SP, Kraus KH, Goldberg VM, Kadiyala S. The effect of implants loaded with autologous mesenchymal stem cells on the healing of canine segmental bone defects. J Bone Joint Surg Am. 1998;80:985-96.

8. Bruder SP, Kurth AA, Shea M, Hayes WC, Jaiswal N, Kadiyala S. Bone regeneration by implantation of purified, culture-expanded human mesenchymal stem cells. J Orthop Res. 1998:16:155-62.

9. Livingston TL, Gordon S, Archambault M, Kadiyala S, McIntosh K, Smith A, et al. Mesenchymal stem cells combined with biphasic calcium phosphate ceramics promote bone regeneration. J Mater Sci Mater Med. 2003;14:211-8.

10. Devine MJ, Mierisch CM, Jang E, Anderson PC, Balian G. Transplanted bone marrow cells localize to fracture callus in a mouse model. J Orthop Res. 2002;20:1232-9.

11. Gaur T, Lengner CJ, Hovhannisyan H, Bhat RA, Bodine PV, Komm BS, et al. Canonical WNT signaling promotes osteogenesis by directly stimulating Runx2 gene expression. J Biol Chem. 2005;280:33132-40.

12. Kim SO, Na HS, Kwon D, Joo SY, Kim HS, Ahn Y. Bone-marrow-derived mesenchymal stem cell transplantation enhances closing pressure and leak point pressure in a female urinary incontinence rat model. Urol Int. 2011:86:110-6.

13. Kawada H, Fujita J, Kinjo K, Matsuzaki Y, Tsuma M, Miyatake H, et al. Nonhematopoietic bone marrow cells can be mobilized and differentiate into cardiomyocytes after myocardial infarction: Possible contribution of mesenchymal stem cells. Blood. 2004;104:3581-7.

14. Hashemi SM, Ghods S, Kolodgie FD, Parcham-Azad K, Keane M, Hamamdzic $D$, et al. A placebo controlled, dose-ranging, safety study of allogenic mesenchymal stem cells injected by endomyocardial delivery after an acute myocardial infarction. Eur Heart J. 2008;29:251-9.

15. Imanishi Y, Saito A, Komoda H, Kitagawa-Sakakida S, Miyagawa S, Kondoh H, et al. Allogenic mesenchymal stem cell transplantation has a therapeutic effect in acute myocardial infarction in rats. J Mol Cell Cardiol. 2008;44:662-71.

16. Hall B, Dembinski J, Sasser AK, Studeny M, Andreeff M, Marini F. Mesenchymal stem cells in cancer: Tumor-associated fibroblasts and cell-based delivery vehicles. Int J Hematol. 2007;86:8-16.

17. Shirley D, Marsh D, Jordan G, McQuaid S, Li G. Systemic recruitment of osteoblastic cells in fracture healing. J Orthop Res. 2005;23:1013-21.

18. Caplan Al. Why are MSCs therapeutic? New data: new insight. J Pathol. 2009;217:318-24
19. Mahmood A, Lu D, Lu M, Chopp M. Treatment of traumatic brain injury in adult rats with intravenous administration of human bone marrow stromal cells. Neurosurgery. 2003;53:697-702. discussion 702-703.

20. Murphy JM, Fink DJ, Hunziker EB, Barry FP. Stem cell therapy in a caprine model of osteoarthritis. Arthritis Rheum. 2003;48:3464-74.

21. Le Blanc K. Immunomodulatory effects of fetal and adult mesenchymal stem cells. Cytotherapy. 2003;5:485-9.

22. Kassem M, Abdallah BM. Human bone-marrow-derived mesenchymal stem cells: biological characteristics and potential role in therapy of degenerative diseases. Cell Tissue Res. 2008;331:157-63.

23. Koc ON, Day J, Nieder M, Gerson SL, Lazarus HM, Krivit W. Allogeneic mesenchymal stem cell infusion for treatment of metachromatic leukodystrophy (MLD) and Hurler syndrome (MPS-IH). Bone Marrow Transplant. 2002;30:215-22.

24. Le Blanc K, Tammik L, Sundberg B, Haynesworth SE, Ringden O. Mesenchymal stem cells inhibit and stimulate mixed lymphocyte cultures and mitogenic responses independently of the major histocompatibility complex. Scand J Immunol. 2003;57:11-20.

25. Block GJ, Ohkouchi S, Fung F, Frenkel J, Gregory C, Pochampally R, et al. Multipotent stromal cells are activated to reduce apoptosis in part by upregulation and secretion of stanniocalcin-1. Stem Cells. 2009;27:670-81.

26. Chen L, Tredget EE, Wu PY, Wu Y. Paracrine factors of mesenchymal stem cells recruit macrophages and endothelial lineage cells and enhance wound healing. PLoS One. 2008:3, e1886.

27. Karp JM, Leng Teo GS. Mesenchymal stem cell homing: the devil is in the details. Cell Stem Cell. 2009;4:206-16.

28. McFarlin K, Gao X, Liu YB, Dulchavsky DS, Kwon D, Arbab AS, et al. Bone marrow-derived mesenchymal stromal cells accelerate wound healing in the rat. Wound Repair Regen. 2006;14:471-8.

29. Zhang T, Lee YW, Rui YF, Cheng TY, Jiang XH, Li G. Bone marrow-derived mesenchymal stem cells promote growth and angiogenesis of breast and prostate tumors. Stem Cell Res Ther. 2013;4:70.

30. Cole JH, van der Meulen MC. Whole bone mechanics and bone quality. Clin Orthop Relat Res. 2011;469:2139-49.

31. Henriques C, Henriques-Pons A, Meuser-Batista M, Ribeiro AS, de Souza W. In vivo imaging of mice infected with bioluminescent Trypanosoma cruzi unveils novel sites of infection. Parasit Vectors. 2014;7:89.

32. Schmidt EV, Christoph G, Zeller R, Leder P. The cytomegalovirus enhancer: a pan-active control element in transgenic mice. Mol Cell Biol. 1990;10:4406-11.

33. Falanga V, Iwamoto S, Chartier M, Yufit T, Butmarc J, Kouttab N, et al. Autologous bone marrow-derived cultured mesenchymal stem cells delivered in a fibrin spray accelerate healing in murine and human cutaneous wounds. Tissue Eng. 2007;13:1299-312.

\section{Submit your next manuscript to BioMed Central and take full advantage of:}

- Convenient online submission

- Thorough peer review

- No space constraints or color figure charges

- Immediate publication on acceptance

- Inclusion in PubMed, CAS, Scopus and Google Scholar

- Research which is freely available for redistribution 